\title{
Renal Hemorrhage, CTCAE
}

National Cancer Institute

\section{Source}

National Cancer Institute. Renal Hemorrhage, CTCAE. NCI Thesaurus. Code C56544.

A disorder characterized by bleeding from the kidney. 